\title{
An RFID-based Point and Listen Interface Providing Library Access for the Visually Impaired
}

\author{
Daniel Fallman ${ }^{1}$, Kent Lindbergh ${ }^{1}$, Oskar Fjellstrom ${ }^{1}$, \\ Lars Johansson $^{1}$, Fredrik Nilbrink ${ }^{1}$, Linda Bogren ${ }^{1}$ \\ ${ }^{1}$ Umeå Design Research Group, Umeå Institute of Design, \\ Umeå University, SE-90187, Umeå, Sweden \\ $\{$ daniel.fallman, kent.lindberg, oskar.fjellstrom, \\ lars.johansson, fredrik.nilbrink, linda.bogren \}@dh.umu.se
}

\begin{abstract}
We present the AudioIndex prototype, a light-weight mobile RFIDbased point-and-listen interface that allows visually impaired to browse and search for audio books within a public library without the need for library staff guidance. AudioIndex has been specifically designed to fit well into the library environment and its routines, a fit made possible by carefully considering interaction styles and by solving a number of technical design challenges, including distribution of data, hardware platform, and RFID specifications.
\end{abstract}

Keywords: Pointing Interface, RFID, Mobile, Wireless, Visually Impaired, Speech Synthesis, Design

\section{Introduction}

The use of RFID (Radio Frequency Identification) tags and readers has increased rapidly over the last years, showing ingenuity in terms of technological development and application. Most RFID systems deployed in library environments however, tend to focus mainly on solving logistical issues, such as automated book check-outs and returns, designed to save time and money. In contrast to these efforts, the AudioIndex project presented in this paper is an attempt at using RFID for something quite different - as a means for improving library accessibility for visually impaired people. The AudioIndex prototype is a light-weight mobile RFID-based point-and-listen interface that allows visually impaired to browse and search for audio books within a public library without the need for library staff guidance.

While public access to library services on a high level can be regarded as essential for society, as literacy and free access to information are fundamental conditions for anyone seeking to participate in the democratic process, the incentives for this project comes from the fact that a number of people do not have access to libraries in the same way as most people do. A particularly vulnerable group is those that due to limitations in their cognitive or physical abilities have no or limited access to library services, including the visually impaired. Throughout this project, we have been working in close collaboration with end users and representatives from the Swedish 
Disability Federation, and their feedback has continuously been used in the implementation process to guide design decisions.

\section{Project Overview}

The Audio Index prototype system has been implemented in eight copies that are currently in operation and undergoing long-term public use and testing at Umeå City Library, Umeå, Sweden. In this paper, we will mainly focus on various aspects of the technical implementation of these prototypes. The prototypes are the result of a larger accessibility design project that has been carried out in four stages between 2002 and 2006, described briefly below. For a more detailed description of this process, see [4].

In the first phase, we compiled existing guidelines for improving accessibility for the visually impaired and carried out field studies and interviews. Based on the information thus gathered, we provided recommendations to the library for enhanced accessibility to its services. These recommendations were specifically tailored to cater to the needs of people with various kinds of cognitive and physical disabilities but would in fact improve library accessibility for all library visitors.

In a second phase, we employed creative group methods and techniques such as brainstorming to generate ideas for increased accessibility to libraries especially for visually impaired visitors. These efforts resulted in a final concept and use scenariowhich the prototype implementation reported in this paper is based on - in which visually impaired users employ the system to browse for audio books by pointing directly with their index fingers on the spine of the book as it stands in the shelf, drawing on similar interaction styles in other domains [3]. Recognizing which book it is the system very quickly provides the user with auditory information about the author, the title, and a summary of the contents of that particular book.

Third, before committing to development of a fully functional system we developed a Wizard-of-Oz prototype to be able to test and evaluate the concept with users early on in a real library environment. Intended users were thus allowed to experience using of the system, with a small amount of invested effort from our side. The results of these tests provided positive feedback from the intended users and a number of ideas for improvement.

Fourth, from these tests, we set out to develop a finalized prototype system in eight copies that were installed in Umeå City Library in March 2006 and is now in full operation.

\section{Prototype Implementation Process}

The finalized AudioIndex prototype was developed over the course of about a year. Compared to typical research prototypes, there were a number of considerations. First, as the system is to be used by the general public on a day-to-day basis, the system has to be robust and fail-safe both when it come to its physical design as well as to its software implementation. Second, as the system will be managed by librarians, with limited knowledge about Bluetooth, RFID, and wireless LAN 
technologies, it also has to be easy to recharge, trouble-shoot, and reset. Third, as we were to make eight copies of the system, it had to be reasonable in terms of the cost of each device.

The part of the AudioIndex prototype that is utilized by the primary user, the library visitor, consists of three interconnected physical parts. First, the main deviceworn around the neck using straps - contains a Dell Axim X30 PDA. The PDA is however fully enclosed in a custom-made plastic container, thus not being exposed to the user. Second, an earpiece provides the auditory information to the user. Volume is controlled using a small control attached to the cable. Third, a small pointing device is worn on the index finger. It consists of a custom-made plastic cover holding a battery and a dismantled MicroSensys iID PEN, connecting wirelessly using Bluetooth to the main device. The infrastructure of the system is largely hidden from the primary users, consisting of a SQL database, a wireless LAN, RFID tags, and a device that facilitates entry of information into the database.

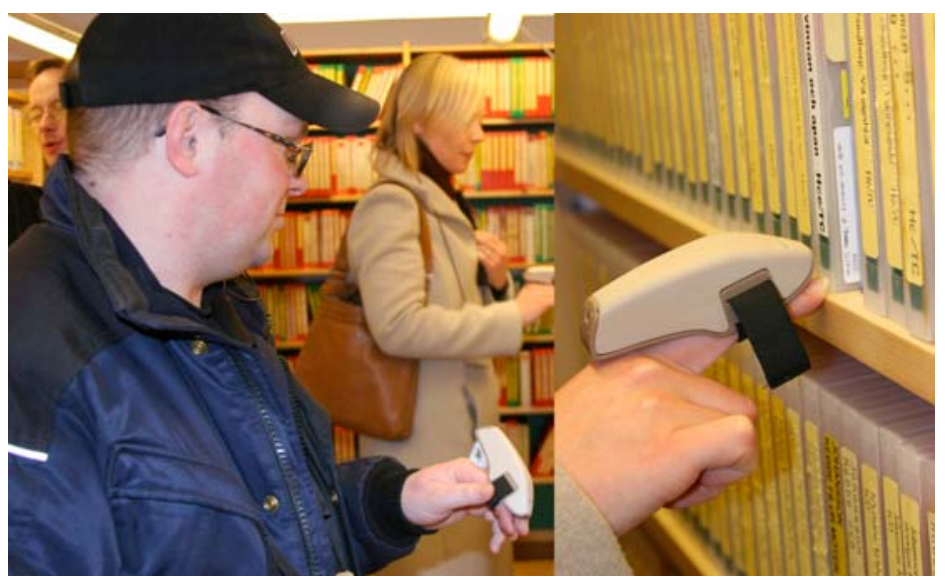

Fig. 1. The AudioIndex prototype in use at Umeå City Library, Umeå, Sweden

\subsection{Deployment environment}

The system was to be installed and used at the Umeå City Library, a public facility with around 2000 visitors per day housing an audio book section that contained about 3500 titles in DAISY format [2]. A DAISY book physically consists of a CD in a plastic case $(20 \times 171 \times 144 \mathrm{~mm})$. When delivered to the library, a DAISY book is fitted with stickers on the front and on the spine that provide information about the book in printed text and sometimes in Braille. It is also fitted with a barcode that is used for logistical purposes.

The entire public area of the library is served by a wireless LAN (WLAN). After the end of the project the test units were to remain in the library and be handled by the library staff, which would also have to maintain the database since the number of DAISY books grows by 20-30 volumes per week. 


\section{Hardware and Software Implementation}

As the user points at the spine of a specific audio book, the RFID reader in the fingermounted pointing device reads the identification number of the iCode-type RFID tag embedded in the book's spine and sends it to the PDA using Bluetooth. The PDA then sends a request over wireless LAN to a SQL server asking for all data for that particular book ID. After receiving this data, the PDA device employs speech synthesis software [5] to produce an audio stream that is fed to the user through the earpiece in the order of author, title, and summary. The entire system has been implemented in C\# using Microsoft Visual Studio .NET 2003.

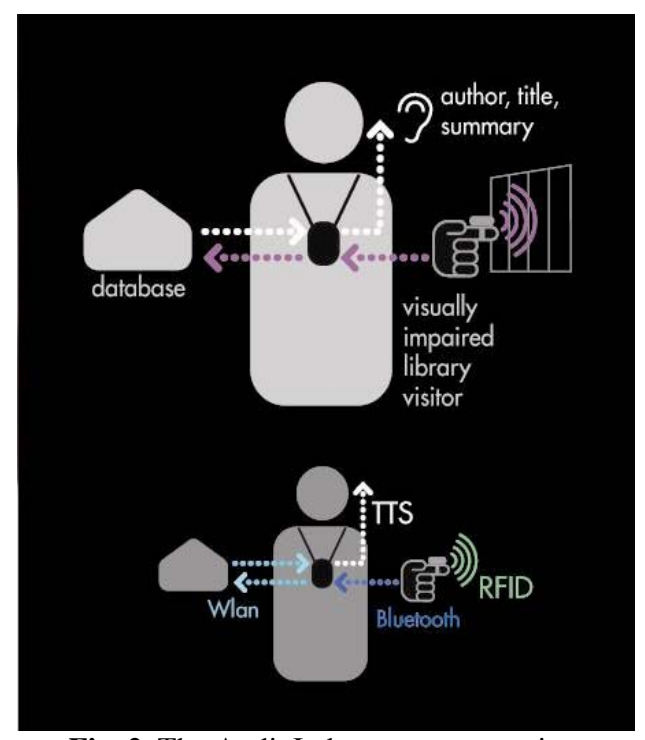

Fig. 2. The AudioIndex system overview

\subsection{RFID Requirements}

We wanted an RFID system that was small enough to fit the problem domain. The objects to be tagged were DAISY books as described above and the tag had to fit in the spine of the book $(20 \times 171 \mathrm{~mm})$. For a visually impaired person the sense of touch takes on some of the tasks that the sense of vision performs for a fully sighted person. An important feature of the design of the reader is therefore that it can be worn on the index finger to allow users to use their fingertips as they would normally do. Therefore the reader needed to be fairly small. The spatial organization of the tagged objects; plastic cases with a $20 \mathrm{~mm}$ wide spine, side by side; set another requirement: that the reader would be able to discriminate the tag at which it is pointed from all other nearby tags. Some RFID readers are unable to read any tag if several tags are within the reading range simultaneously, other readers report all tags that are within the range. We obviously only wanted the tag that was pointed at to be read. 
As far as possible, we also wanted to comply with any current or future standards for RFID use in Swedish libraries, and since we were going to deploy eight systems, the cost-per-device was also a limiting factor.

To deal with these requirements, we chose the Philips ICODE SLI protocol since ongoing discussions in Sweden regarding standardization of RFID in libraries point towards a solution heavily influenced by Danish standards, where ICODE SLI is the chosen RFID standard. We chose the MicroSensys iID ${ }^{\circledR}$ PEN bt reader because this was the only reader available at the time that was small enough, wireless, and supported the ICODE SLI protocol. The tags chosen were I-code SCID PS from the German distributor Pavcard. The tags have 384 bits of free memory, a maximum reading range of $10 \mathrm{~cm}$ and support for anti-collision. What were more important to us were the dimensions of the tags: $25 \times 9 \times 0.3 \mathrm{~mm}$, a low enough silhouette to make it possible to place them on the inside of the spines, thus making the whole solution more robust. Another important feature of these tags was that they were self adhesive.
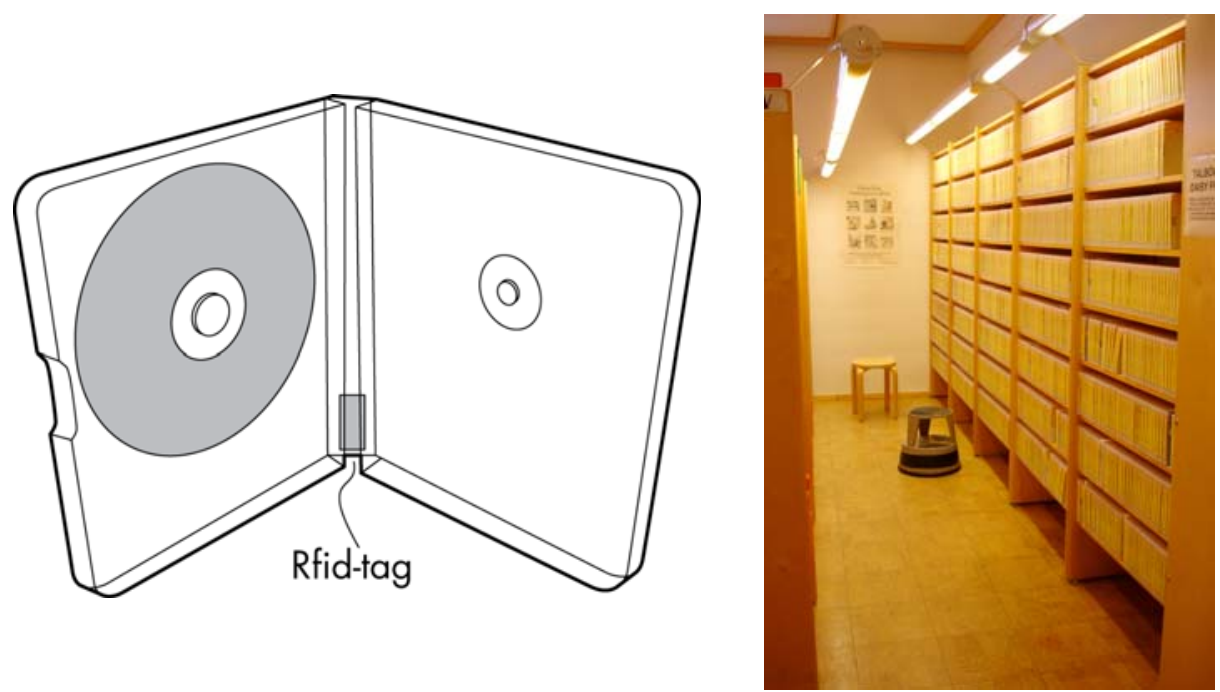

Fig. 3. The tagged object, a plastic case containing a CD (left); Spatial organization of tagged objects (right)

\subsection{Distribution of information}

There were three options to choose from when deciding where to store the actual information content of the system. The first option was to store information about each audio book on the attached RFID tag. This option was quickly discarded since the information that would be presented to the user typically had a length of more than 400 characters per object (not counting overhead information necessary for subdividing data). In some cases the information exceeded 500 characters in length. 384 free bits would not nearly suffice unless we employed some compression 
algorithm, and with such a strategy we would still probably be left with hardly any space left for future additions to the data set.

The second option would be to deploy identical local databases on all PDAs. This would theoretically minimize the time to retrieve information from the database since the database resides on the same device that the retrieve requests originate from. However, this also creates a synchronization problem since the data set grows by about 20 to 30 audio books per week. The eight units would not be docked to a single computer since they needed to be distributed over the library premises and be handled by different librarians. It would have been possible to write code that would copy the most up-to-date database from a central server to each PDA at start-up time. We chose not to do this for a number of reasons. Neither the design team nor the test users experienced such a latency from the moment that a tag was read to the moment when the information was spoken that we felt it necessary to invest project resources to achieve a potentially lower latency. Throughout the project we have used user experience rather than absolute numbers as the meter by which to gauge the performance of our solutions.

The server side of the system needed to be deployed onto a server provided by the municipality for organizational reasons. This meant we had to comply with guidelines set by the IT-department of the municipality. These guidelines restricted our freedom in terms of software solutions and deciding not to write any server side code made it possible to steer clear of the thorn-field of writing fully compliant code.

The third and chosen option was to implement the database as a very simple SQL database containing only one table with seven columns. Whenever information needs to be retrieved from the database a request is sent via WLAN and the response is then returned.

\subsection{Power considerations}

A typical visit to the audio book section does not last more than 30 minutes but we strove to maximize the time a unit could be used. The PDAs were fitted with a high capacity battery (1700 mAh compared to $950 \mathrm{mAh}$ of the out-of-the-box battery) and by programming we turned off the screen of the PDA as soon as the PDA had established contact with the finger worn reader and the SQL database. We did not conduct an exhaustive test of battery capacity but after two hours of continuous use the battery meter indicated that it still held $50 \%$ of its capacity. Our tests with real users indicate that two hours of continuous use is enough for our purposes.

\subsection{Creating the new database}

Creating and maintaining the database would be the responsibility of the library staff but we were responsible for finding a suitable hardware and software solution. Knowing that the number of audio books to be entered into the database was 3500 we realized that each second per book that we could save during the input process would be very useful for the library staff. Therefore, we spent a fair amount of time coming up with a solution that would automate as much of the work as possible. We built a 
custom reading unit that contains a barcode reader and an RFID reader placed in such a way that when an audio book is inserted, the barcode and the RFID code are read and used in a search-and-enter operation that only requires user involvement in special cases when something goes wrong. The typical scenario is that the operator attaches an RFID tag to the inside of the book spine, inserts the book into the reading unit, waits a second or so until information about the book and a message about successful insertion into the database are displayed on the screen. When the book is removed, the information is also removed from the GUI. When dealing with several books, the library staff typically first attaches tags to a pile of books, then inserts them one after the other into the reading unit.

The key to getting hold of the desired information is the barcode, a subpart of which is a seven digit code identifier that we use to search an online database and from that retrieve information about author, title, summary, year of publication, etc.

\subsection{Interaction}

There are two roles, the library staff and the library visitor. When a library visitor wishes to use an AudioIndex unit, the librarian activates the finger worn reader by pressing a small button on its side, resets the PDA and then waits for the PDA to establish contact with both the reader and the database. When this contact has been established, the screen of the PDA is turned off and a red LED on the reader starts blinking. The librarian then inserts the PDA into its case which is worn like a necklace, and attaches the audio cable from the headphones. When the unit has been handed over to the visitor, he or she only has two interaction possibilities; point-andlisten or control the audio volume by turning a wheel on the necklace. When the visitor is done using the unit, the librarian must charge the batteries of both the PDA and the reader in order to ensure that the next visitor will be able to use a fully charged unit.

\section{Discussion}

An important, overall design goal of this project has been to hide interactional as well as computational complexity from the user. The only means by which the user interacts is actually by pointing at things in the physical library environment to get an audio description about the object, or, whenever the user wishes, immediately silence any current audio stream by pointing at the main device itself-all other screens, knobs, and buttons that make up the system are hidden from the user.

Due to the constraints that the system would have to fit into the informational infrastructure of the library and the local municipality, as well as into the daily routines of the library staff, we understood the importance of keeping the project grounded in the reality of the library and its staff. Many implementation decisions have been influenced by and directly related to the necessity of keeping the system realistic in its application context. This is not a bad thing however, since our aim in this project has not been to display our technical prowess but rather create a service 
that would benefit the library and its visitors today, without the need of a co-present research team facilitating its use. The challenges in such a project are different compared to those encountered in technology-driven explorative projects, but not less interesting. Some of the questions and challenges that we have dealt with in this project are discussed below:

Why create a new database instead of piggybacking on the existing database? The library already had a database containing most of the information that we needed to use during the project. Therefore, we explored the possibilities of adding two missing columns to the database; one containing RFID codes and one containing the book summaries. This proved futile for two reasons; it was not technically possible to extend the database and the copyright for the summaries did not allow that information to be added to library database. We were however allowed to use the summaries in a new project database separate from the library database.

Why strive for wireless operation? Wires tend to become rats' nests and thus become hard to handle even for visually enabled people. In our case, some of the librarians as well as the end users were visually impaired, so ease of use through wireless operation had high priority. In the end, we had to settle for headphones connected via wire to the PDA since we experienced severely impaired WLAN connectivity when using Bluetooth headphones. No detailed analysis of this phenomenon was made due to time limitations.

Why not build custom hardware ourselves? Time was a limiting factor, so instead of the to HCI design proverbial process of inventing a slightly different wheel, we opted for off-the-shelf hardware components, thus sacrificing optimal size in favor of time.

Additionally, to duplicate a prototype system tends to be quicker if the hardware consists largely of off-the-shelf products. Replacing faulty parts are likewise made significantly easier by using standard hardware-which is even more true if such an operation is to be performed by someone else than the researchers who built the system.

Why use WLAN to communicate with SQL server? The Umeå City Library already had a good WLAN infrastructure so it was natural to use that resource instead of building another kind of network, e.g. based on Bluetooth.

Why use a PDA? Other options could have been a mobile phone or a miniature computer like Oqo. At the time of the prototype project inception (early 2005) UMPC-grade computers had not been launched. Ideally, we would obviously have wanted to do away with the PDA altogether and include speech synthesis and WLAN capabilities in the finger worn device itself, but since such a product was not readily available and we did not have time or inclination to construct that product within the frames of this project, we wanted to get the smallest possible device that would suit our technical requirements within a reasonable budget. When decisions regarding hardware were also made at a time when there were no mobile phones available with 
WLAN, a speech synthesis that was accessible to third part developers, suitable Bluetooth profiles, and an IDE (integrated development environment) with a low learning threshold. We also had previous experience of developing PDA applications and knew that we did not really have time to spend learning a new IDE.

Why use speech synthesis rather than pre-recorded speech? Why was the speech synthesis done on the PDA? Pre-recorded speech would have given the users an improved experience both in terms of usability and in terms of pleasure. Speech synthesis is generally still not as pleasant to listen to or as easily understood as human speech [cf. 5]. However, since there were no such audio files available, we would have had to create these ourselves and, subsequent to the installation, the library staff would have to spend considerable time creating such audio files. Also, such a system would in the long run be impractical to maintain, requiring new recordings to be made for every added item.

Speech synthesis software is executed on the PDA to avoid high latencies in the delivery of spoken information from the time of pointing. It was hard to estimate the load on the WLAN that was used for a number of other purposes and to minimize the load we opted for delivery of text from the database and conversion to speech on the PDA device rather than streaming audio which would induce a higher load on the limited resources provided by the WLAN.

Why use the synthesized voice 'Ingmar'? 'Ingmar' is the most widely used synthesized voice in Sweden so we expected users, who often use speech synthesis on a daily basis, to feel familiar with Ingmar. This was also a sentiment that was voiced by test users throughout the project. We evaluated several Swedish synthesized voices but found that the one that was most accepted was Ingmar. Subsequently to the installation of AudioIndex in March 2006, another, more life-like voice has become possible to use on PDA; Erik.

Why not make it possible to dynamically alter the speed of the speech synthesis? Many experienced users of speech synthesis technology become so adept at interpreting the voices that it is possible to increase the speed of the speech by as much as $50 \%$ in some cases. Once these users have gotten used to a higher speed, the normal speech synthesis speed seems excruciatingly slow.

In most applications containing speech synthesis it is therefore wise to provide a mechanism by which the user can alter the speed as he/she sees fit. As discussed above, we however wanted to keep the interaction simple so we did not really want to add anything to what we already had, i.e. the ability to point at objects to receive information about those objects and the possibility to control audio volume through a small control wheel on the necklace holding the PDA. Therefore we decided to not let the user alter the speech synthesis speed dynamically but instead be able to choose a higher speed when he/she borrows the unit from the library. This was implemented by us actually hard-coding a slightly higher speech synthesis speed $(20 \%$ higher than normal) into some of the units. While possibly not an ideal solution, at least users are able to tell the library staff whether they want a high or normal speed unit. An alternative design could have been to provide a 'configuration table' with Braille text next to the library desk, allowing users to customize their device by pointing. 


\section{Conclusions}

We have presented the AudioIndex prototype, part of a larger effort in making public libraries more accessible to visually impaired. It allows users to browse and search for audio books without the need for library staff guidance. AudioIndex is a light-weight mobile system based on a combination of using RFID, PDA, and wireless technologies, and allow users to point at objects (typically audio books and bookshelves) and get audio feedback (typically in the form of synthesized speech).

This project challenged the design team in several ways. First, the parts of the system exposed to the users needed to be robust and easy to operate since the target group was varied in terms of age, background and most other characteristics apart from being visually impaired in some way, though such impairments can also be quite different and hence be more or less impairing. This requirement was fulfilled by disallowing any other interaction with the system apart from pointing at objects (typically audio books and bookshelves) and listening at the associated information. Hiding electronic parts of the system inside audio book covers and injection molded plastic casings also minimized the risk of unintentional damages to more delicate parts of the system. Second, the system had to fit into the present library environment both physically and in terms of routines and informational architecture. RFID protocols were chosen so as to harmonize with emerging Swedish library standards for RFID and the construction and maintenance of the system infrastructure utilized existing solutions wherever possible or, if not possible, was designed in such a way that it would minimize interference with other routines. Third, the cost of development was an issue, especially since we were to deploy eight copies of the end user devices. We chose to work with hardware and software modules that required only slight tweaking to provide the desired functionality and focused our own efforts on creating the software, digital glue if you will, that tied all the pieces together to form a whole system.

Acknowledgements. Thanks to Louise Maniette and Anna Karin Bergkvist.

\section{References}

1. Bradley, N.A. \& Dunlop, M.D.: An Experimental Investigation into Wayfinding Directions for Visually Impaired People, Personal and Ubiq. Comp. 9(6) (2005)

2. DAISY: Digital Accessible Information System (DAISY) Consortium. From the World Wide Web: http://www.daisy.org/ (Retrieved February 13, 2007)

3. Fallman, D.: Wear, Point, and Tilt, Proc. of DIS2002 (London, UK, June 25-28) New York, NY: ACM Press (2002)

4. Fallman, D. \& Lindbergh, K.: Inclusive design by taking special measures - making libraries accessible for all, Proceedings of Include 2007 (Royal College of Arts, London, UK, April 2-4). (2007)

5. Pitt, I.J. \& Edwards A.D.N.: Improving the usability of speech-based interfaces for blind users, ACM Conf. on Assistive Technologies (Vancouver, Canada) New York, NY: ACM Press. (1996) 